\title{
OBSERVATORIO
}

\section{LOS GRANDES TEMAS RELACIONADOS CON LA GESTIÓN DE DOCUMENTOS: DESAFÍOS Y OPORTUNIDADES}

\section{Carlota Bustelo-Ruesta}

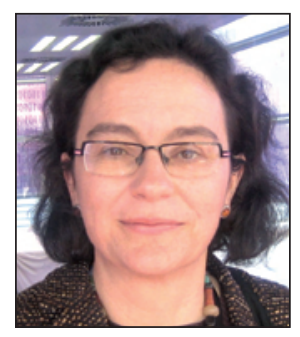

Carlota Bustelo-Ruesta tiene más de 25 años de experiencia en la organización de la información y los documentos tanto en el sector público como en el privado, principalmente en España y Latinoamérica. Desde 2011 ejerce la consultoría de forma independiente, después de haber sido socia directora de dos empresas líderes en el sector de los servicios documentales en España: Inforárea (1996-2010), Gabinete de Asesores Documentalistas (1984-1996). Se ha especializado en la aplicación de tecnologías informáticas al tratamiento de la información y la documentación y tiene una especial experiencia en la integración de los requisitos documentales y de gestión de la información en los procesos de negocio. Es presidenta del comité técnico de Aenor CTN50/SC1 Gestión de Documentos y Aplicaciones y jefa de la delegación española en el subcomité ISO Archive/records management (TC46/SC11). Participa habitualmente en foros profesionales mediante conferencias, artículos, etc. Miembro de ThinkEPI.

Tel.: +34-655853406 carlota@carlotabustelo.com http://www.carlotabustelo.com

\section{Resumen}

La gestión electrónica de la documentación administrativa en las organizaciones se enfrenta a importantes desafíos: integración de los procesos y controles documentales en los procesos de trabajo rutinarios, análisis de riesgos, técnicas y controles necesarios para la gestión de documentos y evidencias digitales (metadatos, firma, autenticidad, integridad, preservación), y restricción o no del acceso por parte de los ciudadanos o clientes. Se invita a que los profesionales de la información tomen un papel activo en la compleja resolución de tales problemáticas.

\section{Palabras clave}

Retos, Archivos, Gestión de documentos, Registros digitales, Metadatos, Profesionales, Firma electrónica, Preservación, Tendencias, Evolución, Documentos electrónicos.

Title: The big issues related to document and record management: challenges and opportunities

\begin{abstract}
The electronic management of documents and records is facing major challenges: the integration of document processes and checks in routine work processes; risk analysis; techniques and controls needed to manage records and digital evidence controls (metadata, digital signature, authenticity, integrity, preservation); and access restrictions for citizenship or customers. Information professionals are invited to take an active role in resolving these complex problems.
\end{abstract}

\section{Keywords}

Challenges, Archives, Record management, Document management, Digital records, Metadata, Professionals, Preservation, Trends, Evolution, Electronic records.

Bustelo-Ruesta, Carlota. "Los grandes temas relacionados con la gestión de documentos: desafíos y oportunidades". El profesional de la información, 2011, marzo-abril, v. 20, n. 2, pp. 129-133.

DOI: 10.3145/epi.2011.mar.01

\section{Introducción}

Siempre que escribo o hablo sobre estos temas me siento en la obligación de aclarar qué entiendo por "gestión de documentos". En este Observatorio me voy a referir a la gestión de la información creada y recibida por una organización en el transcurso de sus actividades y el desarrollo de sus funciones. En algunos sectores profesionales la expresión se está consolidando, pero no creo que pueda decirse que es una expresión entendida por todos. El mismo problema de entendimiento ha llevado a algunos colegas franceses, después de un debate terminológico con otros países francófonos, a proponer el uso de "gestion des informations et des documents d'activité" para referirse a este campo. El tiempo dirá si han tenido éxito. 


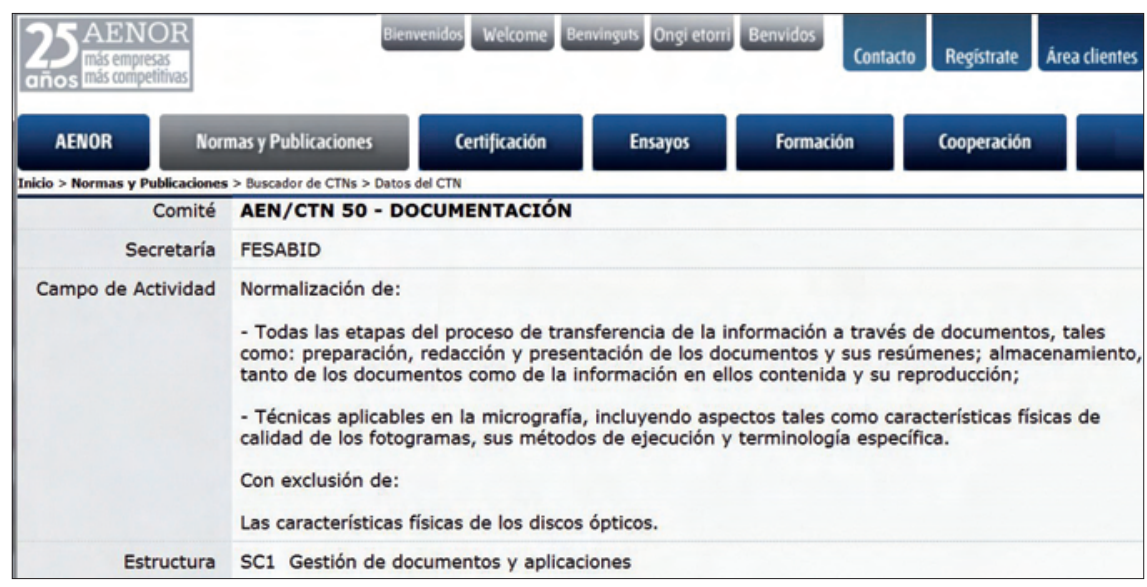

Asociación Española de Normalización y Certificación, http://www.aenor.es

Cuando leí por primera vez el tema de éste número de EPI "archivos administrativos e intranets" lo identifiqué con la gestión de documentos tal como he definido en el párrafo anterior. Pero me asaltó una gran duda ¿podemos/debemos seguir hablando de archivos administrativos?

El entorno digital y la profunda trasformación de nuestras organizaciones han dejado el término archivos administrativos algo caduco

Entendiendo que la razón principal de esta expresión es la distinción con los archivos históricos, pero creo que el entorno digital y la profunda trasformación que se está imponiendo en nuestras organizaciones y en nuestras vidas han dejado esta denominación algo caduca. Es un término que está asociado indisolublemente a los documentos en papel. Todavía nos queda un largo recorrido para que en nuestras organizaciones no existan documentos en papel, pero los cimientos están puestos y la velocidad es de vértigo. Es difícil que alguien asocie los grandes proyectos de gestión de documentos y expedientes electrónicos con los archivos administrativos. Los primeros se han posicionado como elementos estratégicos para el desarrollo de la e-administración o el e-business, mientras los segundos se visualizan como elementos operativos que no queda más remedio que mantener. El lenguaje es un elemento poderoso de estructuración del pensamiento y de la misma forma que hemos dejado de decir "viejos" para adoptar la denominación de "tercera edad", evitando las connotaciones despectivas del primer término, la sustitución de determinadas expresiones o denominaciones nos ayudan a "vendernos" mejor en el terreno profesional.

Desde este Observatorio voy a resaltar los cuatro grandes temas que desde mi punto de vista son los más importantes de la gestión de documentos (electrónicos) en este momento. Todos ellos representan desafíos para los profesionales que nos dedicamos a esto,

\section{International} Organization for Standardization pero al mismo tiempo abren grandes oportunidades para el desarrollo de nuestro trabajo.

\section{Integración de los procesos y controles documentales en los procesos de trabajo}

La integración de los procesos y controles documentales en los procesos de trabajo debe ser el objetivo principal de cualquier modelo de gestión de documentos. En organizaciones donde la inmediatez y la efectividad a golpe de tecla son los valores más apreciados, las personas que desempeñan los distintos procesos de trabajo automatizados no deben ser conscientes de que al mismo tiempo que hacen su trabajo están haciendo gestión documental. Mientras realizan las tareas que les han sido asignadas, los documentos necesarios se crean con sus metadatos correspondientes, se clasifican de forma correcta y se les aplican las políticas corporativas de acceso y conservación.

Nuestro gran desafío es trabajar conjuntamente con los que diseñan la automatización de procesos y las aplicaciones que interactúan con clientes y administrados para que los requerimientos de la buena gestión de documentos se integren en las aplicaciones y en la arquitectura tecnológica. Nuestra oportunidad es dejar de ser vistos como meros ejecutores (personas que hacen carpetas, las meten en cajas, describen los documentos y los encuentran cuando se les piden) para formar parte de los estrategas que definen los requerimientos de la organización y supervisan el cumplimiento de los mismos.

\section{Deben ser los mismos trabajadores de la organización los que desempeñando las distintas tareas estén haciendo automá- ticamente la gestión documental}

\section{Análisis de riesgos}

No estamos acostumbrados a trabajar con los conceptos de análisis de riesgos, pero en la gestión de documentos es un

International Standards for Business Government and Society

\begin{tabular}{l|lll} 
Home & Products & Standards development & News and media
\end{tabular}

Standards development $>$ Technical committees $>$ Other bodies developing standards

Processes and procedures

Technical committees

Business plans

International Organization for Standardization, http://www.iso.org

\section{TC 46/SC 11}

Archives/records management 
componente esencial que nos permite la toma de decisiones adecuadas. Existen al menos dos niveles de evaluación y gestión de riesgos en relación con la gestión de documentos:

- El nivel estratégico que nos hace evaluar cuáles son los riesgos de una organización si no gestiona adecuadamente los documentos que son la evidencia de sus actuaciones. Por ejemplo: ¿Qué consecuencias tiene que una universidad no encuentre los expedientes de sus alumnos?

- El nivel operativo que nos hace evaluar los riesgos del mal funcionamiento de los distintos procesos de gestión documental. Por ejemplo: ¿Qué consecuencias tiene guardar los documentos en formatos obsoletos?

Hay algunos intentos de relacionar el análisis de riesgos con la gestión de documentos, pero aún no se ha establecido una metodología ampliamente aceptada. Es de esperar que en los próximos años éste sea uno de los aspectos que más se desarrolle acercando la gestión de documentos a las técnicas de gestión de riesgos utilizadas en otros ámbitos de la gestión de las organizaciones y el management.

\section{Técnicas y controles necesarios para la gestión de documentos y evidencias digitales}

Desde el punto de vista técnico los documentos electrónicos nos plantean nuevos desafíos que tenemos que resolver para poder gestionar los mismos durante todo el tiempo que sea necesario. Sin depreciar en absoluto las sólidas bases que representan los principios archivísticos y de gestión de documentos, existen tres puntos de atención que requieren un mayor desarrollo conceptual y práctico:

Con los documentos electrónicos la verdadera gestión documental es la de los metadatos

\section{a. Metadatos}

Hablando de documentos electrónicos la gestión de los metadatos se convierte en la verdadera gestión documental. Si entendemos como documentos electrónicos los ficheros que contienen información (un pdf, un excel, una imagen, un vídeo, un registro de base de datos, un sms, etc.), y denominamos metadatos a toda la información que es necesaria para gestionarlos, nos damos cuenta que cualquier proceso documental es en realidad un proceso de captura, gestión y explotación de metadatos.
Los fácilmente identificables son los que nos permiten contextualizar el documento en sí y sin los cuales sería imposible entender de qué trata. Pero también son metadatos la información que nos permite clasificar los documentos en una estructura predefinida, limitar o expandir el acceso y uso, identificar las acciones de disposición o preservación previstas para uno determinado o recoger los eventos que han sucedido a un documento a lo largo del tiempo.

Y cuando se trata de cómo relacionar esta información (metadatos) y como gestionarla integrándose en los sistemas de información de las organizaciones, es cuando damos el salto y nos damos cuenta que no es posible hacer gestión de documentos montando un sistema de información independiente del resto, sino que la gestión de documentos debe preverse en cualquier plan de sistemas, arquitectura de información, etc. Los límites no existen y el desafío de la implementación práctica no es algo de mañana sino de hoy.

España es una referencia mundial en la implantación de los certificados digitales

\section{b. Firmas y autenticidad}

España es una referencia mundial en la implantación de los certificados digitales y la firma avanzada certificada por una autoridad tercera, y sin embargo para la gestión de los documentos electrónicos las firmas se han convertido en uno de los grandes problemas a resolver. A mi modo de ver una interpretación muy estrecha de las posibilidades de la tecnología unida a una interpretación muy concreta de lo que será la legalidad (basadas ambas en la imaginación de un escenario de futuro y no en un escenario ya existente) está llevando a implantaciones tecnológicamente muy complicadas y costosas, basadas en refirmas de documentos, donde es difícil por ahora encontrar un beneficio justificable.

Probablemente en el punto actual lo que sucede con las firmas avanzadas comprobadas por una autoridad tercera es que están dando cumplimiento a dos de las características que debe tener un documento:

- Autenticidad, mediante la comprobación de un tercero.

- Integridad, mediante la realización de un hash (algoritmo para identificar y representar un documento mediante una clave o código).

Si sólo se reconoce esta fórmula para conseguir estas características en un documento, acabaremos concluyendo que todos los documentos han de ser firmados con certificado digital aunque sean documentos internos, lo cual es cuando menos un derroche de
International Records Management Association, http://www.arma.org/

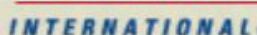

\section{ARMA International is a not-for-profit association and your source for authoritative education, the latest legislative updates, standards \& best practices. \\ The Authority on Managing Records \& Information}




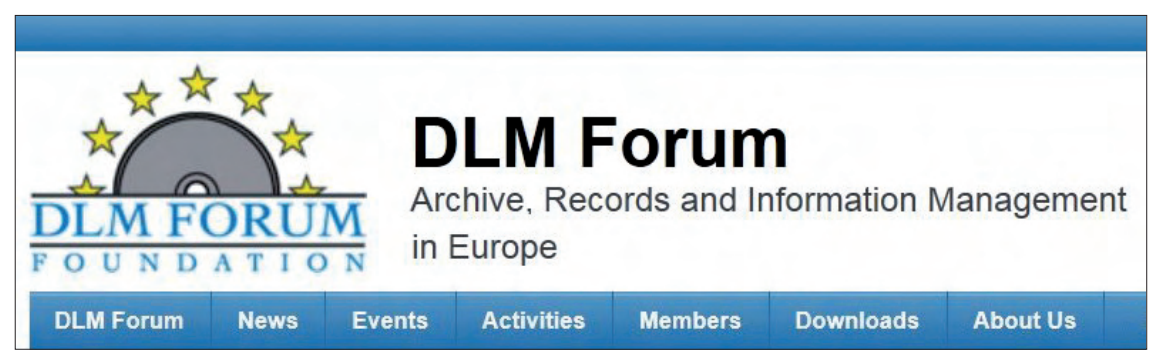

Document Lifecyle Management Forum, http://www.dlmforum.eu

recursos. Los especialistas en gestión documental podemos proponer reconocer la autenticidad de documentos internos basándose en los usuarios y contraseñas y establecer mecanismos que aseguren la integridad de todos los documentos con independencia de su autenticidad basándose en la restricción de permisos, el audit log (registro de accesos para controlar cambios) y en procedimientos internos que pueden incluir tanto soluciones de hardware como de software.

Al mismo tiempo debemos ser conscientes que la única validación de la firma desde el punto de vista de la autenticidad que necesitamos requerir es aquella que se produce en el momento de la misma firma. Yo hoy puedo actuar como Ministro de Justicia, pero mañana me pueden cesar, lo que no invalida mi firma de ayer.

Como gestionar esto a lo largo del tiempo es uno de los grandes retos de hoy, del que no veremos resultados hasta mucho más adelante. Las soluciones que se propongan deben tener en cuenta el largo plazo, cuya cuantificación difiere radicalmente si hablamos con un profesional de la información o con un informático. Y además en este punto las opiniones de los abogados también son esenciales. Nuestro papel es contribuir con todos ellos a buscar soluciones (que están muy lejos de estar normalizadas y consolidadas), no vale con esperar a que otros decidan y simplemente acatar órdenes.

Nuestro papel es contribuir a buscar soluciones; no vale con esperar a que otros decidan y simplemente acatar órdenes

\section{c. Preservación digital: conversión y migración}

Aunque no sabemos cómo resolver la contradicción con el punto anterior (los documentos firmados al ser convertidos perderían su integridad), todos sabemos que la información electrónica puede perderse muy fácilmente. Quien más y quien menos tiene una anécdota que contar a este respecto

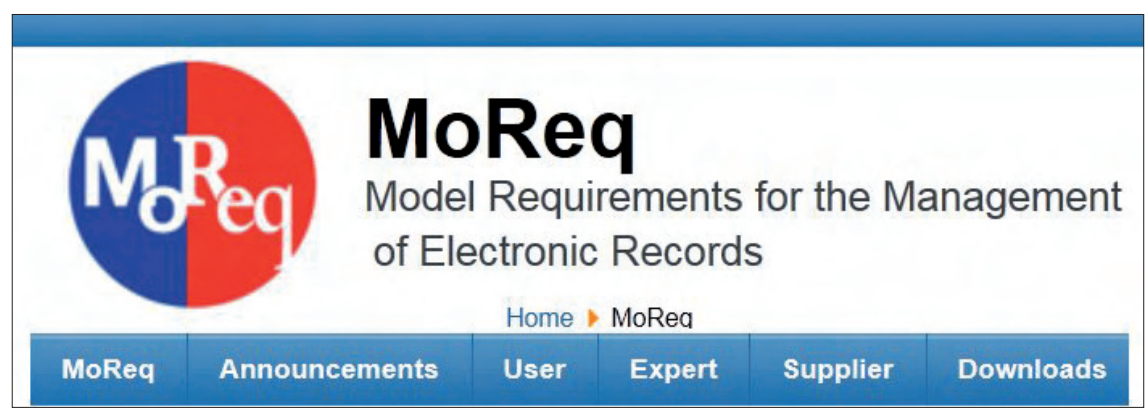

Model Requirements for the Management of Electronic Records, http://www.dlmforum.eu/... y todos somos conscientes de que hay mucha información perdida referente a los años de la explosión de la tecnología.

Esto hace que la preservación, entendida como las medidas preventivas a llevar a cabo para evitar la obsolescencia y destrucción de los documentos, haya pasado a tener un papel muy importante. Altamente relacionada con la gestión de riesgos, en este momento se consolidan y normalizan los aspectos más funcionales de la misma dando por hecho que la tecnología permite la conversión y migración (todos los días miles de documentos nacidos en otros formatos son convertidos a pdf, por poner sólo un ejemplo), como la planificación, la validación y la documentación.

\section{Existen herramientas de redaction que permiten ocultar información de un do- cumento electrónico}

\section{Acceso en la e-sociedad}

La sociedad se debate en cómo conjugar el derecho a la información y la transparencia (de los ciudadanos sobre las actuaciones de sus administraciones, de los accionistas sobre lo que sucede en la compañía, de los clientes sobre sus proveedores, de todos sobre las actuaciones que afectan al medio ambiente, etc.) con la protección fundamentalmente de la intimidad de las personas y otras restricciones.

Esta discusión no es sólo del entorno digital, pero éste la acentúa debido a las enormes posibilidades que nos ofrece internet para el acceso a la información.

La idea central es que el acceso a la información puede realizarse de forma directa sin que tenga que existir intervención humana que evalúe, determine y entregue o deniegue la información. Esta intervención humana no tiene que confundirse con la identificación de las personas que acceden a la información, que siempre es posible por medios totalmente automatizados.

Sin embargo hay mucha gente que defiende que la intervención humana también es necesaria en un entorno automatizado y nos alertan de los peligros de abrir la información en la Red.

La construcción de plataformas de acceso a documentos con complicados sistemas de seguridad y acceso que intenten resolver hasta el más pequeño caso de conflicto es una tarea ardua y complicada. Herramientas de redaction que permiten ocultar información de un documento electrónico tienen su nicho de mercado.

El gran debate está servido y las soluciones y decisiones pueden apoyarse en distintos ejes: 
- El puramente tecnológico, con funcionalidades que nos permitan sofisticadas acciones en el acceso a la información.

- El conceptual o de base, proponiendo nuevas formas de confección de documentos. Por ejemplo, si hay información que no puede difundirse no incluirla en documentos que sí deben ser accesibles.

- El legal o de conflicto de derechos, estableciendo los límites y marco de actuación. www.digitalpreservationeurope.eu

\section{digital preservation $\mathcal{C}$ urope}

addressing digital memory preservation challenges

home \& news|contact us | site map | rss | search | staff

Digital Preservation Europe (DPE), http://www. digitalpreservationeurope.eu

Los profesionales de la gestión de documentos tenemos que ser parte activa en este debate, insistiendo una y otra vez en que cualquiera que sea la restricción de acceso que se aplique a determinados documentos debe ser limitada en el tiempo.

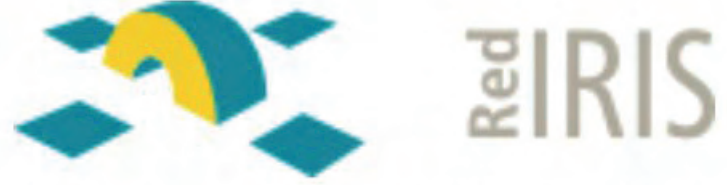

http://www.rediris.es/list/info/iwetel.html

\section{IWETEL}

Foro para profesionales de bibliotecas y documentación

\section{IweTel, foro de información y debate de la biblioteconomía y la documentación}

Fundada por Tomàs Baiget en 1993, IweTel es la lista pionera en español de los profesionales de las bibliotecas, documentación, bases de datos y sistemas de información en general.

Al principio se alojó en Sarenet y en 1998 pasó a RedIRIS. Posteriormente se han ido creado otras listas más especializadas como Arxiforum (archivos), Bib-Med (información bio-médica), Bescolar (bibliotecas escolares), Incyt (indicadores científicos), etc., pero IweTel, con más de 5.000 miembros, es la lista de referencia, el medio de comunicación básico y central para los profesionales de la información.

En la lista se cumple la conocida regla del $80 / 20$ (el $80 \%$ de los mensajes los genera el $20 \%$ de los inscritos), o su reciente reformulación a 90, 9, 1\%: el 90\% de los inscritos son pasivos, casi nunca envían nada, el 9\% (unos 360) participa alguna vez, y existe un 1\% (50 personas) que genera la mayoría de mensajes.

Con el aumento de inscritos y el número de mensajes (algunas semanas se distribuyen más de 100) fue necesario hacer la lista moderada, y en ello estamos los 4 firmantes, intentando aplicar nuestro sentido común para decidir cuáles se aprueban y cuáles no, y evitando los mensajes repetidos. Rechazamos alrededor de un 15-20\%, lo cual a veces provoca quejas de sus autores, y para dirimir las dudas se creó un Consejo Asesor formado por veteranos de la lista, a quienes los moderadores pedimos consejo.

La lista cumple los dos objetivos básicos típicos: tablón de anuncios (conferencias, cursos, publicaciones, noticias) y foro de debates. Además se usa como sistema abierto de evaluación por pares (open peer review) de las notas que los miembros del think tank ThinkEPI envían periódicamente a la lista para su pública crítica y discusión. Esas notas y los principales mensajes que generan se publican cada año re-editados en el Anuario ThinkEPI de la editorial EPI.

Con los cambios tecnológicos habidos a lo largo de estos años y, más recientemente, con las nuevas plataformas web 2.0, se ha planteado muchas veces si las listas de correo se han hecho "obsoletas". La verdad es que pensamos que una lista sigue siendo el medio ideal de comunicación de una comunidad profesional: rápida, limpia, discreta y eficaz, lejos de la faramalla de las redes sociales, también muy interesantes y útiles pero para otras cosas.

Más información e inscripciones:

http://www.rediris.es/list/info/iwetel.html

Javier Leiva-Aguilera (Catorze.com), Paco López-Hernández (Universidad Carlos III de Madrid), Isabel Olea (Universidad de León) y Tomàs Baiget (EI profesional de la información). 


\section{doc6 en 30 segundos}

\section{Nuestra misión}

Aportar soluciones para mejorar la eficiencia de empresas y organizaciones a través de una eficaz gestión de la información y del conocimiento.

\section{La empresa}

Creada en 1988. Le aportamos más de $\mathbf{2 0}$ años de experiencia.

a Consultoría en gestión documental, de la información y del conocimiento

- Sistemas de Gestión Documental: mapas documentales, cuadros de clasificación de archivos, concreción de funcionales, etc.

a Intranets, Extranets. Arquitectura y usabilidad web. Taxonomías

- Servicios profesionales adecuados a cada necesidad

a Soluciones en gestión documental

a Recursos electrónicos de información. Representante en España de OCLC

a Formación personalizada

\section{Los 6 valores de doc6}

Los valores por los que se rige la actividad de doc6 definen nuestro código ético, nuestros métodos de trabajo y organización, la cultura de la empresa. Son los siguientes:

axcelencia en calidad

a Innovación permanente. Creatividad

a Cooperación y trabajo en equipo

a Confidencialidad

- Profesionalidad y honestidad con clientes y proveedores

Responsabilidad social

\section{Los clientes}

El principal activo de la empresa, junto con sus empleados.

Más de $\mathbf{1 0 0 0}$ clientes: empresas, laboratorios, administraciones públicas, universidades, hospitales, bufetes de abogados, museos, fundaciones, colegios profesionales.

\section{Nuestros profesionales}

Un equipo de más de $\mathbf{1 0 0}$ profesionales.

Una eficaz suma de conocimientos: informáticos y documentalistas con experiencia.

El $90 \%$ son licenciados superiores o diplomados en informática o documentación. 